\title{
A novel analytic potential function applied to neutral diatomic molecules and charged lons
}

\author{
Chang-Feng Yu, Chang-Jun Zhu, Chong-Hui Zhang, Li-Xun Song, Qiu-Pin Wang
}

Department of physics, School of Science, Xi'an Polytechnic University, Xi’an, China; yuh55@126.com

Received 24 November 2009; revised 8 January 2010; accepted 30 January 2010.

\begin{abstract}
In this paper, a new method on constructing analytical potential energy functions is presented, and from this a analytical potential energy function applied to both neutral diatomic molecules and charged diatomic molecular ions is obtained. This potential energy function includes three dimensionless undetermined parameters which can be determined uniquely by solving linear equations with the experimental spectroscopic parameters of molecules. The solutions of the dimensionless undetermined parameters are real numbers rather than complex numbers, this ensures that the analytical potential energy function has extensive universality. Finally, the potential energy function is examined with four kinds of diatomic molecules or ions-homonuclear neutral diatomic molecule $\mathrm{H}_{2}\left(\mathbf{X}^{1} \Sigma_{\mathrm{g}}^{+}\right), \mathrm{K}_{\mathbf{2}}\left(\mathbf{B}^{1} \prod_{\mathrm{u}}\right)$ and $\mathrm{Li}_{\mathbf{2}}\left(\mathbf{B}^{1} \prod_{\mathrm{u}}\right)$, homonuclear charged diatomic molecular ion $\mathrm{He}_{2}^{+}\left(\mathrm{X}^{2} \Sigma_{\mathrm{u}}^{+}\right), \mathbf{N}_{2}^{+}\left(\mathrm{X}^{2} \Sigma_{\mathrm{g}}^{+}\right)$and $\mathrm{O}_{2}^{+}\left(\mathrm{X}^{2} \Pi_{\mathrm{g}}\right)$, heternuclear neutral diatomic Molecule $\operatorname{AIBr}\left(A^{1} \Pi\right)$, $\operatorname{PuO}\left(X^{1} \Sigma_{g}^{+}\right)$and $\mathrm{NaLi}\left(X^{1} \Sigma_{g}^{+}\right)$, heternuclear charged diatomic Molecular ion $\operatorname{BC}^{-}\left(X^{3} \Pi\right)$, $\mathrm{MgH}^{+}\left(\mathrm{X}^{1} \Sigma^{+}\right)$and $\mathrm{HCl}^{+}\left(X^{2} \prod_{i}\right)$, as a consequence, good results are obtained.
\end{abstract}

Keywords: Diatomic Molecules And lons; Potential Energy Function; Force Constants; Spectroscopic Parameters; Phase Factor

\section{INTRODUCTION}

Analytical potential energy functions are of great significance in the study of material science, molecular spectrum, reaction dynamics of atoms and molecules, vibrational and rotational energy-level structures of molecules, interactions between laser and matter, photoionization etc. [1-3] Due to the importance and extensive applications of the potential energy function, the corresponding research works have been carried on all along [4-6]. So far, the representative analytical potential energy function proposed have Morse potential [7], Rydberg potential [8], Murrell-Sorbie potential (M-S) [9] and Huxley-Murrell-Sorbie potential (HMS) [10] etc.

Recently, Sun Weiguo et al have proposed an energy consistent method (ECM) and constructed a new physically well behaved analytical potential function of a diatomic system called ECM potential [11]. These potential functions above have merits and defects respectively, they are valid in describing the behaviors of some individual or classificatory diatoms and molecules. But none of them can describe both neutral diatomic molecules and charged diatomic molecular ions and describe precisely the behaviors of potential energy function over the whole range of internuclear distance. Seen from expressional forms, most of these potential energy functions adopt the forms of ploynomial and exponential. In this paper, a cosine function with a phase factor is used as basic potential energy function and, through renormalization to the phase factor, a universal potential energy function applied to four kinds of diatomic molecules or ions - homonuclear neutral diatomic molecules, homonuclear charged diatomic molecular ions, heternuclear neutral diatomic molecules and heternuclear charged diatomic molecular ions is given. Finally, the potential energy function is examined with twelve different kinds of diatomic molecules and ions etc., as a consequence, good results are obtained.

\section{FUNDAMENTAL SUPPOSITIONS, AND DERIVATION OF A UNIVERSAL ANALYTIC POTENTIAL FUNCTION}

Suppose that the potential function of diatomic molecular satisfies the following relation

$$
\begin{aligned}
V(r) & =A \cos \psi(r)+B \\
\text { where } \psi(r) & =\varphi+\arccos (\rho / r)
\end{aligned}
$$

where, $A, B$ are undetermined constants, $\psi(r)$ is a 
phase factor related to $\phi, \rho$ and the internuclear distance $r$, here $\phi$ is equivalent phase difference between two interacting atoms, $\rho$ is equilibrium internuclear distance. Substituting Eq.2 into Eq.1, yields

$$
\begin{array}{r}
V(r)=A \cos (\varphi+\arccos (\rho / r))+B \\
=A\left((\rho / r) \cos \varphi-\sqrt{1-\rho^{2} / r^{2}} \sin \varphi\right)+B
\end{array}
$$

Eq.3 is a basic analytical potential energy function. In order to obtain the universal analytical potential function of diatomic molecules and ions, renormalization should be needed for the term $\sqrt{1-\rho^{2} / r^{2}}$ in Eq.3, so as to ensure that the derivatives of each order of the $\mathbf{E q . 3}$ are continuous and finite at equilibrium distance $r=\rho$. Thus we can expand the term into binomial series

$$
\sqrt{1-\rho^{2} / r^{2}}=-\sum_{i=0}^{\infty} \frac{(2 i) !}{4^{i}(i !)^{2}(2 i-1)}\left(\frac{\rho}{r}\right)^{2 i}
$$

Here, Eq.4 is a infinite series, it need to be truncateed into finite terms and its following infinite terms should be absorbed into three undetermined coefficients $a, b, c$, so from Eq.4, we have

$$
\begin{gathered}
\sqrt{1-\rho^{2} / r^{2}} \approx-\left\{\sum_{i=0}^{n} \frac{(2 i) !}{4^{i}(i !)^{2}(2 i-1)}\left(\frac{\rho}{r}\right)^{2 i}+a\left(\frac{\rho}{r}\right)^{2 n+2}\right\} \\
=-\left\{\sum_{i=0}^{n} H(i)\left(\frac{\rho}{r}\right)^{2 i}+a\left(\frac{\rho}{r}\right)^{2 n+2}+b\left(\frac{\rho}{r}\right)^{2 n+4}+c\left(\frac{\rho}{r}\right)^{2 n+6}\right\} \\
\text { where } H(i)=\frac{(2 i) !}{4^{i}(i !)^{2}(2 i-1)}
\end{gathered}
$$

Generally, the potential energy function satisfies asymptotic condition $\lim _{r \rightarrow \infty} V(r)=0$, so from Eq.3

$$
\text { we have } B=A \sin \phi
$$

Substituting Eq.5 and Eq.7 into Eq.3, and notice $H(0)=-1$, yields

$$
\begin{aligned}
V(r) & =\frac{A \rho}{r} \cos \phi+A \sin \phi\left\{\sum_{i=1}^{n} H(i)\left(\frac{\rho}{r}\right)^{2 i}\right. \\
& \left.+a\left(\frac{\rho}{r}\right)^{2 n+2}+b\left(\frac{\rho}{r}\right)^{2 n+4}+c\left(\frac{\rho}{r}\right)^{2 n+6}\right\}
\end{aligned}
$$

In Eq.8, the undetermined constant $A$ can be determined according to the properties of potential energy function. At the equilibrium distance $r=\rho$, the potential value is equal to the negative value of dissociation energy $D_{e}$, i.e. $V(\rho)=-D_{e}$, and the first derivatives of $V(r)$ with respect to $r$ is zero.

So from Eq.8, we obtain

$$
\begin{aligned}
& V(\rho)=A \cos \phi+A \sin \phi\left\{\sum_{i=1}^{n} H(i)+a+b+c\right\}=D_{e} \\
& \left(\frac{\mathrm{d} V}{\mathrm{~d} r}\right)_{r=\rho}=-\frac{A}{\rho} \cos \phi-\frac{A}{\rho} \sin \phi\left\{\sum_{i=1}^{n} H(i)(2 i)\right. \\
& +a(2 n+2)+b(2 n+4)+c(2 n+6)\}=0
\end{aligned}
$$

From Eq.9 and Eq.10, the solutions of $A$ and $\cos \phi$ can be given as follows

$$
\begin{gathered}
A=\frac{D_{e}}{\sin \phi}\left(\sum_{i=1}^{n} H(i)(2 i-1)+a(2 n+1)+b(2 n+3)+c(2 n+5)\right)^{-1} \\
\cos \phi=-\sin \phi\left\{\sum_{i=1}^{n} H(i)(2 i)+a(2 n+2)\right. \\
+b(2 n+4)+c(2 n+6)\}
\end{gathered}
$$

Substituting Eq.11 and Eq.12 into Eq.8, yields

$$
\begin{gathered}
V(r)=D_{e}\left\{\sum_{i=1}^{n} H(i)(\rho / r)^{2 i}+a(\rho / r)^{2 n+2}+b(\rho / r)^{2 n+4}\right. \\
+c\left(\frac{\rho}{r}\right)^{2 n+6}-\left[\sum_{i=1}^{n} H(i)(2 i)+a(2 n+2)+b(2 n+4)\right. \\
\left.+c(2 n+6)] \frac{\rho}{r}\right\}\left(\sum_{i=1}^{n} H(i)(2 i-1)+a(2 n+1)+\right. \\
b(2 n+3)+c(2 n+5))^{-1}
\end{gathered}
$$

Eq.13 is the universal analytical potential energy function that is required. The undetermined parameters $a, b, c$ can be determined with the experimental spectroscopic parameters $\left(\omega_{e}, \omega_{e} \chi_{e}, \alpha_{e} B_{e}\right)$ of molecules or fitting method using singlepoint potential energy scanning. When $n=1,2,3$, from Eq.13, we have

$$
\begin{aligned}
& V(r)=\left(\frac{2 D_{e}}{1+6 a+10 b+14 c}\right)\left\{\frac{1}{2}\left(\frac{\rho}{r}\right)^{2}+a\left(\frac{\rho}{r}\right)^{4}+\right. \\
& \left.b\left(\frac{\rho}{r}\right)^{6}+c\left(\frac{\rho}{r}\right)^{8}-(1+4 a+6 b+8 c) \frac{\rho}{r}\right\}, \quad(n=1) \\
& V(r)=\left(\frac{8 D_{e}}{7+40 a+56 b+72 c}\right)\left\{\frac{1}{2}\left(\frac{\rho}{r}\right)^{2}+\frac{1}{8}\left(\frac{\rho}{r}\right)^{4}+\right. \\
& \left.a\left(\frac{\rho}{r}\right)^{6}+b\left(\frac{\rho}{r}\right)^{8}+c\left(\frac{\rho}{r}\right)^{10}-\left(\frac{3}{2}+6 a+8 b+10 c\right) \frac{\rho}{r}\right\}
\end{aligned}
$$




$$
\begin{aligned}
& V(r)=\left(\frac{16 D_{e}}{19+112 a+144 b+176 c}\right)\left\{\frac{1}{2}\left(\frac{\rho}{r}\right)^{2}+\frac{1}{8}\left(\frac{\rho}{r}\right)^{4}+\right. \\
& \frac{1}{16}\left(\frac{\rho}{r}\right)^{6}+a\left(\frac{\rho}{r}\right)^{8}+b\left(\frac{\rho}{r}\right)^{10}+c\left(\frac{\rho}{r}\right)^{12}-(15 / 8+8 a+10 b \\
& \left.+12 c) \frac{\rho}{r}\right\} \quad(n=3)
\end{aligned}
$$

\section{USING EXPERIMENTAL SPECTROSCOPIC PARAMETERS TO DETERMINE $a, b, c$}

The undetermined parameters $a, b, c$ can be determined with the experimental spectroscopic parameters $\left(\omega_{e}, \omega_{e} \chi_{e}, \alpha_{e} B_{e}\right)$ of diatomic molecules or ions. The principle of this method is, according to the relationship between undetermined parameters and force constants, to obtain $a, b, c$ by solving linear equations. From Eq.13, the general expression of force constants at the equilibrium internuclear distance can be given as follows

$$
\begin{gathered}
f_{m}=\left(\frac{\mathrm{d}^{m} V}{\mathrm{~d} r^{m}}\right)_{r=\rho}=\frac{(-1)^{m} V_{0}}{\rho^{m}}\left\{\sum_{i=1}^{n}\left[H(i) \prod_{j=0}^{m-1}(2 i+j)\right]+\right. \\
a \frac{(2 n+1+m) !}{(2 n+1) !}+b \frac{(2 n+3+m) !}{(2 n+3) !}+c \frac{(2 n+5+m) !}{(2 n+5) !} \\
\left.-\left[\sum_{i=1}^{n} H(i)(2 i)+a(2 n+2)+b(2 n+4)+c(2 n+6)\right] m !\right\} \\
(m=2,3,4)
\end{gathered}
$$

where

$$
V_{0}=\frac{D_{e}}{\sum_{i=1}^{n} H(i)(2 i-1)+a(2 n+1)+b(2 n+3)+c(2 n+5)}
$$

From Eq.17 and Eq.18, when $n=1,2,3$, the following linear equations can be obtained

$$
\begin{aligned}
& \left\{\begin{array}{l}
\frac{1+12 a+30 b+56 c}{1+6 a+10 b+14 c}=X=\frac{f_{2} \rho^{2}}{2 D_{e}} \\
\frac{2+32 a+100 b+224 c}{1+6 a+10 b+14 c}=Y=-\frac{f_{3} \rho^{3}}{6 D_{e}} \\
\frac{3+62 a+240 b+644 c}{1+6 a+10 b+14 c}=Z=\frac{f_{4} \rho^{4}}{24 D_{e}}
\end{array}\right. \\
& (n=1, \text { and } 62 X-25 Y+4 Z-32 \neq 0)
\end{aligned}
$$

$$
\begin{aligned}
& \left\{\begin{array}{l}
\frac{10+120 a+224 b+360 c}{7+40 a+56 b+72 c}=X=\frac{f_{2} \rho^{2}}{2 D_{e}} \\
\frac{24+400 a+896 b+1680 c}{7+40 a+56 b+72 c}=Y=-\frac{f_{3} \rho^{3}}{6 D_{e}} \\
\frac{43+960 a+2576 b+5640 c}{7+40 a+56 b+72 c}=Z=\frac{f_{4} \rho^{4}}{24 D_{e}}
\end{array}\right. \\
& (n=2, \text { and } 98 X-31 Y+4 Z-80 \neq 0)
\end{aligned}
$$

$$
\begin{aligned}
& \left\{\begin{array}{l}
\frac{35+448 a+720 b+1056 c}{19+112 a+144 b+176 c}=X=\frac{f_{2} \rho^{2}}{2 D_{e}} \\
\frac{98+1792 a+3360 b+5632 c}{19+112 a+144 b+176 c}=Y=-\frac{f_{3} \rho^{3}}{6 D_{e}} \\
\frac{206+5152 a+11280 b+21648 c}{19+112 a+144 b+176 c}=Z=\frac{f_{4} \rho^{4}}{24 D_{e}}
\end{array}\right. \\
& (n=3, \text { and } 142 X-37 Y+4 Z-160 \neq 0)
\end{aligned}
$$

In Eqs.19-21, the relationships between force constants and spectroscopic parameters are as follows

$$
\begin{gathered}
f_{2}=4 \pi^{2} \mu \omega_{e}^{2} c^{2} \\
f_{3}=-\frac{3 f_{2}}{\rho}\left(1+\frac{\alpha_{e} \omega_{e}}{6 B_{e}^{2}}\right) \\
f_{4}=\frac{f_{2}}{\rho^{2}}\left[15\left(1+\frac{\alpha_{e} \omega_{e}}{6 B_{e}^{2}}\right)-\frac{8 \omega_{e} \chi_{e}}{B}\right]
\end{gathered}
$$

The Eqs.19-21 above are all linear equations, when the conditions of $62 X-25 Y+4 Z-32 \neq 0,98 X-31 Y$ $+4 Z-80 \neq 0$ and $142 X-37 Y+4 Z-160 \neq 0$ are satisfied with respect to Eqs.19-21, they have unique real number solutions for the undetermined parameters $a, b, c$. Calculations show that the conditions above are always tenable in general. This ensures that the analytical potential function Eq.13 has extensive universality, which can describe any of diatomic molecules and ions especially the behaviors of molecules near equilibrium internuclear distance. So far, the most extensively used analytical potential energy function is Murrel-Sorbie (M-S) potential. The undetermined parameters in Murrel-sorbie potential which are determined by experimental spectroscopic parameters have no unique solutions and contain complex number solutions. Thus, the M-S potential is extremely limited in applications to some diatomic molecules and ions. [12]

\section{APPLIED EXAMPLES OF THE UNIVERSAL ANALYTICAL POTENTIAL ENERGY FUNCTION}

For examining potential energy function Eq.13, fifty kinds of neutral diatomic molecules and charged diatomic 
Table 1. Experimental spectroscopic parameters of diatomic molecules and ions.

\begin{tabular}{|c|c|c|c|c|c|c|c|}
\hline states & $\omega_{e} / \mathrm{cm}^{-1}$ & $\omega_{e} \chi_{e} / \mathrm{cm}^{-1}$ & $B_{e} / \mathrm{cm}^{-1}$ & $\alpha_{e} / \mathrm{cm}^{-1}$ & $\rho / \mathrm{nm}$ & $D_{e} / \mathrm{eV}$ & Refs. \\
\hline $\mathrm{H}_{2}\left(\mathrm{X}^{1} \sum_{\mathrm{g}}^{+}\right)$ & 4401.21 & 121.34 & 60.809 & 3.062 & 0.0741 & 4.747 & {$[13]$} \\
\hline $\mathrm{K}_{2}\left(\mathrm{~B}^{1} \prod_{\mathrm{u}}\right)$ & 75.00 & 0.3876 & 0.04824 & 0.000235 & 0.4235 & 0.514 & {$[14]$} \\
\hline $\mathrm{Li}_{2}\left(\mathrm{~B}^{1} \prod_{\mathrm{u}}\right)$ & 270.7 & 2.9530 & 0.5770 & 0.0083 & 0.2936 & 0.3700 & {$[15]$} \\
\hline $\mathrm{He}_{2}^{+}\left(\mathrm{X}^{2} \sum_{\mathrm{u}}^{+}\right)$ & 1698.52 & 35.30 & 7.211 & 0.2240 & 0.1080 & 2.475 & {$[16]$} \\
\hline $\mathrm{N}_{2}^{+}\left(\mathrm{X}^{2} \sum_{\mathrm{g}}^{+}\right)$ & 2207.20 & 16.1360 & 1.9320 & 0.0200 & 0.1116 & 6.341 & [14] \\
\hline $\mathrm{O}_{2}^{+}\left(\mathrm{X}^{2} \Pi_{\mathrm{g}}\right)$ & 1905.30 & 16.304 & 1.6905 & 0.0189 & 0.1117 & 6.7792 & [17] \\
\hline $\operatorname{AlBr}\left(\mathrm{A}^{1} \Pi\right)$ & 297.2 & 6.400 & 0.1555 & 0.00216 & 0.2322 & 2.400 & {$[14]$} \\
\hline $\mathrm{PuO}\left(\mathrm{X}^{1} \sum_{\mathrm{g}}^{+}\right)$ & 822.28 & 2.500 & 0.3365 & 0.00146 & 0.1830 & 7.3372 & [18] \\
\hline $\operatorname{NaLi}\left(X^{1} \sum_{g}^{+}\right)$ & 256.80 & 1.610 & 0.3960 & 0.0036 & 0.2810 & 0.8570 & [13] \\
\hline $\mathrm{BC}^{-}\left(\mathrm{X}^{3} \Pi\right)$ & 1301.4 & 9.820 & 1.418 & 0.0155 & 0.1445 & 5.588 & [19] \\
\hline $\operatorname{MgH}^{+}\left(\mathrm{X}^{1} \Sigma^{+}\right)$ & 1226.60 & 16.300 & 3.321 & 0.0640 & 0.16530 & 2.100 & {$[14]$} \\
\hline $\mathrm{HCl}^{+}\left(\mathrm{X}^{2} \prod_{\mathrm{i}}\right)$ & 2675.4 & 53.50 & 9.9463 & 0.3183 & 0.13152 & 4.480 & {$[14]$} \\
\hline
\end{tabular}

Table 2. Potential parameters and force constants of diatomic molecules and ions.

\begin{tabular}{|c|c|c|c|c|c|c|c|c|c|}
\hline states & $D_{e} / \mathrm{eV}$ & $\rho / \mathrm{nm}$ & $n$. & $a$ & $b$ & $c$ & $f_{2} / 10^{2} \mathrm{aJ} \cdot \mathrm{nm}^{-2}$ & $f_{3} / 10^{3} \mathrm{aJ} \cdot \mathrm{nm}^{-3}$ & $f_{4} / 10^{4} \mathrm{aJ} \cdot \mathrm{nm}^{-4}$ \\
\hline $\mathrm{H}_{2}\left(\mathrm{X}^{1} \sum_{\mathrm{g}}^{+}\right)$ & 4.747 & 0.0741 & 1 & -0.4615 & 0.2008 & -0.0367 & 5.752 & -37.43 & 238.7 \\
\hline $\mathrm{K}_{2}\left(\mathrm{~B}^{1} \Pi_{\mathrm{u}}\right)$ & 0.514 & 0.4235 & 3 & -0.64605 & 0.47875 & -0.10628 & 0.0646 & -0.1035 & 0.045 \\
\hline $\mathrm{Li}_{2}\left(\mathrm{~B}^{1} \Pi_{\mathrm{u}}\right)$ & 0.370 & 0.2936 & 3 & -0.69431 & 0.57753 & -0.14667 & 0.1463 & -0.3177 & 0.4546 \\
\hline $\mathrm{He}_{2}^{+}\left(X^{2} \Sigma_{u}^{+}\right)$ & 2.475 & 0.1080 & 3 & -0.8073 & 0.6766 & -0.1729 & 3.401 & -20.97 & 101.23 \\
\hline $\mathrm{N}_{2}^{+}\left(\mathrm{X}^{2} \sum_{\mathrm{g}}^{+}\right)$ & 6.341 & 0.1116 & 3 & -0.5889 & 0.44335 & -0.10575 & 20.11 & -160.61 & 1059.1 \\
\hline $\mathrm{O}_{2}^{+}\left(\mathrm{X}^{2} \Pi_{\mathrm{g}}\right)$ & 6.7792 & 0.1117 & 3 & -0.55136 & 0.37972 & -0.0829 & 17.09 & -142.33 & 918.04 \\
\hline $\operatorname{AlBr}\left(\mathrm{A}^{1} \Pi\right)$ & 2.400 & 0.2322 & 1 & -0.4874 & 0.2759 & -0.0577 & 1.049 & -7.357 & 21.837 \\
\hline $\mathrm{PuO}\left(\mathrm{X}^{1} \sum_{\mathrm{g}}^{+}\right)$ & 7.3372 & 0.183 & 3 & -0.64234 & 0.49584 & -0.11975 & 5.959 & -27.03 & 98.61 \\
\hline $\operatorname{NaLi}\left(X^{1} \sum_{g}^{+}\right)$ & 0.857 & 0.281 & 3 & -0.78369 & 0.67093 & -0.17435 & 0.2095 & -0.4434 & 0.7012 \\
\hline $\mathrm{BC}^{-}\left(\mathrm{X}^{3} \Pi\right)$ & 5.588 & 0.1445 & 3 & -0.6900 & 0.5404 & -0.1317 & 5.677 & -31.49 & 140.50 \\
\hline $\mathrm{MgH}^{+}\left(\mathrm{X}^{1} \Sigma^{+}\right)$ & ) 2.10 & 0.1653 & 3 & -0.74505 & 0.62333 & -0.15926 & 1.6468 & -6.5343 & 19.547 \\
\hline $\mathrm{HCl}^{+}\left(\mathrm{X}^{2} \prod_{\mathrm{i}}\right)$ & 4.480 & 0.13152 & 3 & -0.80085 & 0.66178 & -0.16814 & 4.3433 & -25.61 & 138.1 \\
\hline
\end{tabular}

Table 3. Potential parameters of Murrel-Sorbie potential of diatomic molecules and ions.

\begin{tabular}{|c|c|c|c|c|c|}
\hline states & $a_{1} / \mathrm{nm}^{-1}$ & $a_{2} / \mathrm{nm}^{-2}$ & $a_{3} / \mathrm{nm}^{-3}$ & $\rho / \mathrm{nm}$ & $D_{e} / \mathrm{eV}$ \\
\hline $\mathrm{H}_{2}\left(\mathrm{X}^{1} \sum_{\mathrm{g}}^{+}\right)$ & 39.601 & 405.91 & 3577.1 & 0.0741 & 4.747 \\
\hline $\mathrm{K}_{2}\left(\mathrm{~B}^{1} \prod_{\mathrm{u}}\right)$ & 1.227 & -38.457 & 161.65 & 0.4235 & 0.514 \\
\hline $\mathrm{Li}_{2}\left(\mathrm{~B}^{1} \prod_{\mathrm{u}}\right)$ & 28.79 & 291.03 & 1317.4 & 0.2936 & 0.370 \\
\hline $\mathrm{He}_{2}^{+}\left(X^{2} \Sigma_{u}^{+}\right)$ & 32.363 & 94.792 & 584.08 & 0.108 & 2.475 \\
\hline $\mathrm{N}_{2}^{+}\left(\mathrm{X}^{2} \sum_{\mathrm{g}}^{+}\right)$ & 70.966 & 1528.3 & 15675 & 0.1116 & 6.341 \\
\hline $\mathrm{O}_{2}^{+}\left(\mathrm{X}^{2} \Pi_{\mathrm{g}}\right)$ & 1.376 & 68.968 & 1085.1 & 0.1117 & 6.7792 \\
\hline $\operatorname{AlBr}\left(\mathrm{A}^{1} \Pi\right)$ & 9.1044 & -95.068 & 2072.2 & 0.2322 & 2.400 \\
\hline $\mathrm{PuO}\left(\mathrm{X}^{1} \sum_{\mathrm{g}}^{+}\right)$ & 30.377 & 207.88 & 804.25 & 0.183 & 7.3372 \\
\hline $\operatorname{NaLi}\left(X^{1} \sum_{g}^{+}\right)$ & 19.865 & 121.01 & 329.24 & 0.281 & 0.857 \\
\hline $\mathrm{BC}^{-}\left(\mathrm{X}^{3} \Pi\right)$ & 27.880 & 71.540 & 634.30 & 0.1445 & 5.588 \\
\hline $\mathrm{MgH}^{+}\left(\mathrm{X}^{1} \Sigma^{+}\right)$ & 33.953 & 331.66 & 1450.8 & 0.1653 & 2.10 \\
\hline $\mathrm{HCl}^{+}\left(\mathrm{X}^{2} \prod_{\mathrm{i}}\right)$ & 29.618 & 150.74 & 1133.1 & 0.13152 & 4.48 \\
\hline
\end{tabular}




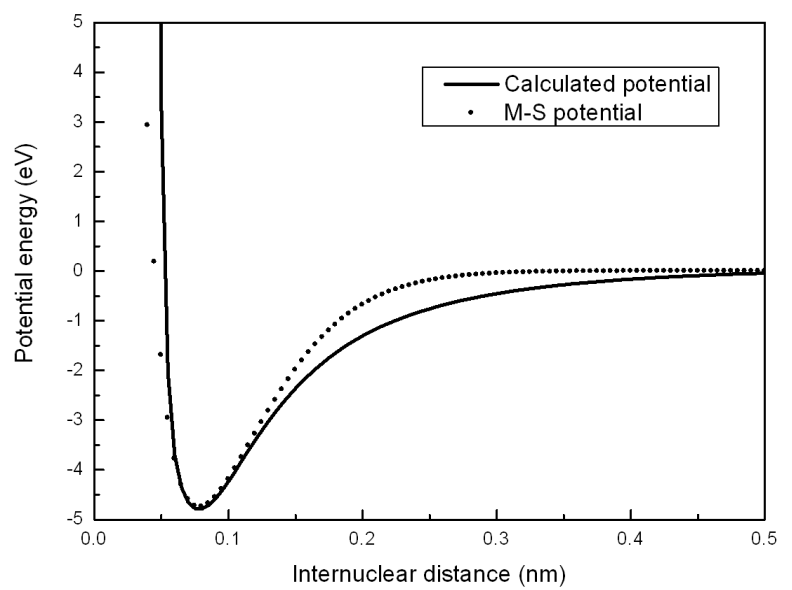

Figure 1. Potential curve of $\mathrm{H}_{2}-\mathrm{X}^{1} \sum_{\mathrm{g}}^{+}$.

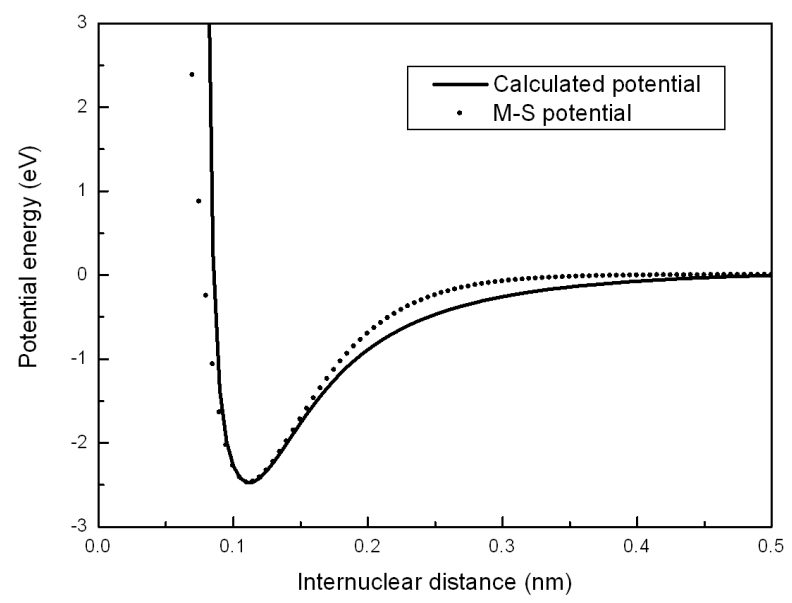

Figure 2. Potential curve of $\operatorname{He}_{2}^{+}\left(X^{2} \Sigma_{u}^{+}\right)$

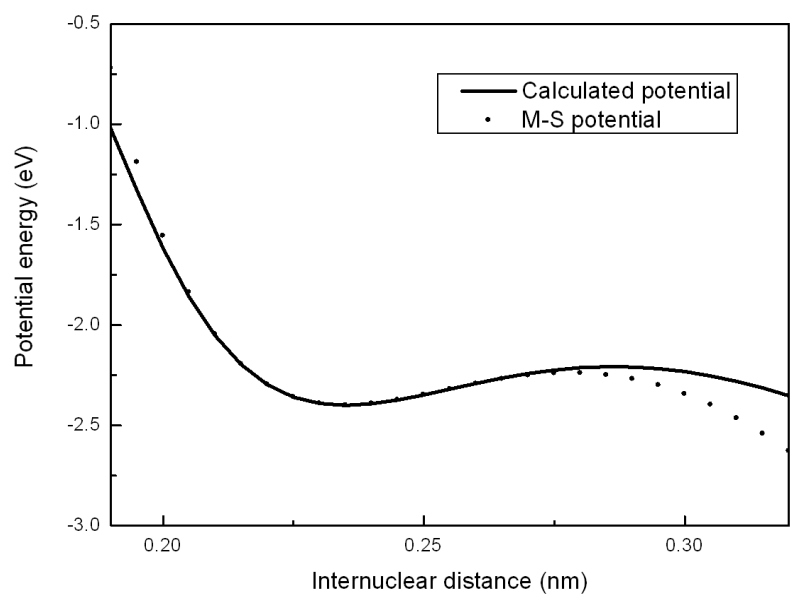

Figure 3. Potential curve of $\mathrm{AlBr}-\mathrm{A}^{1} \Pi$.

molecular ions have ever been investigated and good results are obtained. Calculations show that two common potential energy curves, i.e. steadystate and metastable state

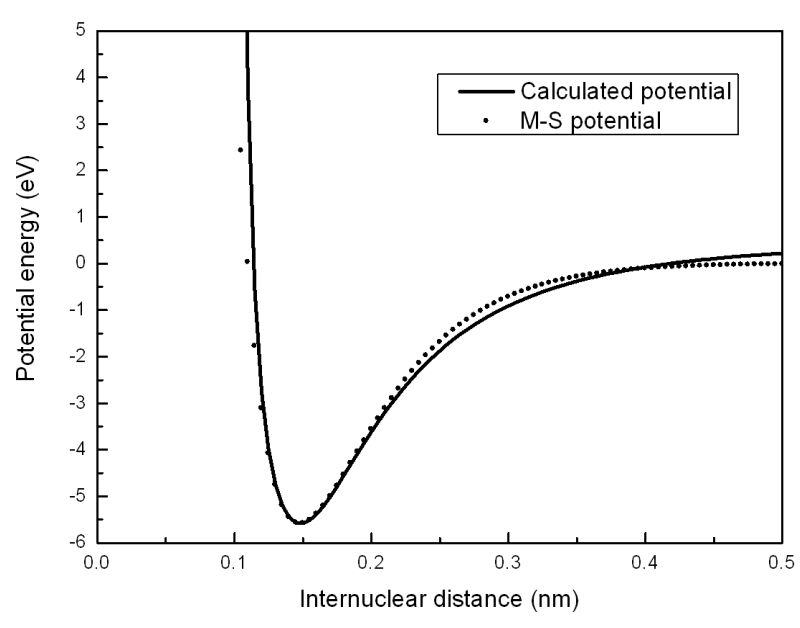

Figure 4. Potential curve of $\mathrm{BC}^{-}\left(\mathrm{X}^{3} \Pi\right)$.

can be given by using the potential energy function determined with experimental spectroscopic parameters. The experimental spectroscopic parameters of $\mathrm{H}_{2}\left(\mathrm{X}^{1} \Sigma_{\mathrm{g}}^{+}\right)$, $\mathrm{He}_{2}^{+}\left(\mathrm{X}^{2} \sum_{\mathrm{u}}^{+}\right), \operatorname{AlBr}\left(\mathrm{A}^{\mathrm{l}} \Pi\right)$ and $\mathrm{BC}^{-}\left(\mathrm{X}^{3} \Pi\right)$ etc. are listed in Table 1. According to Eqs.22-24, the corresponding force constants can be obtained by using the experimental spectroscopic parameters above, and substituting these force constants into Eq.19 or Eq.21, then the undetermined parameters $a, b, c$ can be calculated by solving the linear equations. The calculation values are listed in Table 2. The potential energy curves (to be calculated and plotted by using Eq.14 and Eq.16 directly with Origin 7.0 software) plotted by Eq.14 and Eq.16 of $\mathrm{H}_{2}\left(\mathrm{X}^{1} \sum_{\mathrm{g}}^{+}\right), \mathrm{He}_{2}^{+}\left(\mathrm{X}^{2} \sum_{\mathrm{u}}^{+}\right), \mathrm{AlBr}\left(\mathrm{A}^{1} \Pi\right)$ and $\mathrm{BC}^{-}\left(\mathrm{X}^{3} \Pi\right)$ are illustrated in Figures 1-4. As comparison, in the Figs., the dot lines are the potential curves which are plotted by using the most extensively used Murrel-Sorbie Potential. The M-S potential expression is as follows

$$
\begin{aligned}
V(r)= & -D_{e}\left[1+a_{1}(r-\rho)+a_{2}(r-\rho)^{2}\right. \\
& \left.+a_{3}(r-\rho)^{3}\right] \exp \left[-a_{1}(r-\rho)\right]
\end{aligned}
$$

The relationships between undetermined parameters of M-S potential and force constants are as follows

$$
\begin{gathered}
D_{e}\left(a_{1}^{2}-2 a_{2}\right)=f_{2} \\
2 D_{e}\left(3 a_{1} a_{2}-3 a_{3}-a_{1}^{3}\right)=f_{3} \\
D_{e}\left(3 a_{1}^{4}-12 a_{1}^{2} a_{2}+24 a_{1} a_{3}\right)=f_{4}
\end{gathered}
$$

\section{CONCLUSIONS}

In this paper, we first introduce the phase concept to the studies of analytical potential energy functions and get 
good results. This shows that the method of constructing analytical potential energy function by means of phase is effective and reliable. Compared with other potential energy functions, the potential energy function given in this paper has two merits: 1) The undetermined parameter equations determined by experimental spectroscopic parameters are linear equations. Because these linear equations have unique real number solutions, so this potential energy function has a extensive universality; 2) This potential energy function can describe four different kinds of diatomic molecules or ions-homonuclear neutral diatomic molecules, homonuclear charged diatomic molecular ions, heternuclear neutral diatomic molecules and heternuclear charged diatomic molecular ions; In addition, This potential energy function can also describe accurately the behaviors of potential curves over a fairly wide range of internuclear distance.

Potential energy functions of diatomic molecules are the basis to the studies of multi-atomic molecules, ions and clusters, which have extremely significances and applied values in the study of material science, molecular spectrum chemical reaction etc. Chemical reaction, molecular collision and many other problems need precise analytical potential energy functions. Thus, the studies of analytical potential energy function will still be important subject in atomic and molecular physics.

\section{REFERENCES}

[1] Yiannopoulou, A., Jeung, G.-H., Su, J.P., et al. (1999) Potential-energy curves for highly excited electronic states in diatomic molecules related to the atomic orbital undulations [J]. Physical Review A, 59(2), 1178-1186

[2] Liu, G.Y., Sun, W.G. and Feng, H. (2004) Studies on the analytical potential energy function of diatomic molecular Ion $\mathrm{XY}^{+}$using variational method $[\mathrm{J}]$. Science in China (Series G), 47(2), 154-164.

[3] Maniero, M. and Acioli, P.H. Full configuration interaction pseudopotential determination of the ground-state potential energy curves of $\mathrm{Li}_{2}$ and $\mathrm{LiH}[\mathrm{J}]$. International Journal of Quantum Chemistry, 103(5), 711-717.

[4] Xie, R.H. and Gong, J.B. (2005) A Simple Three- parameter model potential for diatomic systems: From weakly and strong to metastable molecular ions [J]. Physical Review Letters, 95, 263202.

[5] Yu, C.f., Yan, K. and Liu, D.Z. (2006) A universal analytic potential-energy function based on a phase factor [J]. Acta Metallurgica Sinca (English Letters), 19(6), 455-468.

[6] Esteves, C.S., de Oliveira, H.C.B., Ribeiro, L., et al. (2006) Modeling diatomic potential energy curves through the generalized exponential function [J]. Chemical Physics Letters, 427(1), 10-13.

[7] Morse, P.M. (1929) Diatomic molecules according to the wave mechanics. II. Vibrational levels [J]. Physical Review Letters, 34: 57-64.

[8] Rydberg, R. (1931) Graphische Darstellung einiger Bandenspe-ktroskopischer Ergebnisse [J]. Z Physics, 73: 376-385.

[9] Murrel, J.N. and Sorbie, K.S. (1974) New analytic from for the potential energy curves of stable diatomic state [J]. Journal of the Chemical Society, Faraday Trans, II, 70, 1552-1557.

[10] Huxley, P. and Murrel, J.N. (1983) Ground-state diatomic potential [J]. Journal of the Chemical Society, Faraday Trans II, 79, 323-328.

[11] Sun, W.G. and Feng, H. (1999) An energy-consistent method for potential energy curves of diatomic molecules [J]. Journal of Physics B: Atomic, Molecular and Optical Physics, 32(21), 5109-5121.

[12] Zhu, Z.H. and Yu, H.G. (1997) Molecular structure and molecular potential energy function [M]. Science Press, Beijing, 38-123.

[13] Geng, Z.D., Fan, X.W. and Zhang, Y.S. (2006) Structure and potential energy function of the ground state of $X Y$ (H, Li, Na).[J] Acta Physica Sinica, 55(05), 2175-2179.

[14] Herzberg, G. (1983) Molecular spectra and molecular structure (i. spectra of diatomic molecules) [M]. Science Press, Beijing, 397-454

[15] Yu, B.H., Shi, D.H., Sun, J.F., Zhu, Z.L., Liu, Y.F. and Yang, X.D. (2007) Ab initio Calculation on Accurate Analytic Potential Energy Functions and Harmonic Frequencies of $c^{3} \sum_{g}^{+}$and $\mathrm{B}^{1} \prod_{\mathrm{u}}$ States of Dimer $7 \mathrm{Li} 2[\mathrm{~J}]$. Chinese Physics, 16(8), 2371-2377.

[16] Haberland, H., Issendorff, B.V., Frochtenicht, R., et al. (1995) Absorption Spectroscopy and Photodissociation Dynamics of Small Helium Cluster Ions [J] Journal of Chemical Physics, 102(22), 8773-8779.

[17] Sarvpreet, K. and Mahajan, C.G. (1999) Wei hua's four-parameter potential comments and computation of molecular constants_ $\alpha_{e}$ and $\omega_{e} \chi_{e}$ [J]. Pramana journal of physics, 52(4), 409-420.

[18] Gao, T., Wang, H.Y., Yi, Y.-G., Tan, M.-L., Zhu, Z.-H., Sun, Y., Wang, X.-L. and Fu, Y.-B. (1999) Ab intio calculation of the potential energy function and thermodynamic functions for ground state $X^{5} \Sigma^{-}$of $\mathrm{PuO}[\mathrm{J}]$. Acta. Physica Sinica, 48(12), 2222-2227.

[19] Tzeli, D. and Mavridis, A. (2001) Continuing our study on the electronic structure of the carbides $\mathrm{BC}$ and $\mathrm{AlC}[\mathrm{J}]$. Journal of Physical Chemistry, A105, 1175-1184. 